Canadian

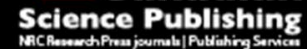

Canadian Journal of Chemistry Revue canadienne de chimie

\title{
Oxo-analogues of 20-hydroxyecdysone in the synthesis of novel fluorinated ecdysteroid derivatives
}

\begin{tabular}{|r|l|}
\hline Journal: & Canadian Journal of Chemistry \\
\hline Manuscript ID & cjc-2017-0629.R1 \\
\hline Danuscript Type: & Article \\
\hline Complete List of Authors: & $\begin{array}{l}\text { Savchenko, Rimma; Institute of Petrochemistry and Catalysis of Russian } \\
\text { Academy of Sciences, } \\
\text { Apaeva, Anastasia; Institute of petrochemistry and catalysis } \\
\text { Kostyleva, Svetlana; Institute of Petrochemistry and Catalysis of Russian } \\
\text { Academy of Sciences } \\
\text { Mozgovoj, Oleg; Institute of Petrochemistry and catalysis } \\
\text { Odinokov, Victor; Institute of Petrochemistry and Catalysis of Russian } \\
\text { Academy of Sciences, } \\
\text { Parfenova, Lyudmila; Institute of Petrochemistry and Catalysis of Russian } \\
\text { Academy of Sciences, }\end{array}$ \\
\hline Is the invited manuscript for \\
consideration in a Special \\
Issue?:
\end{tabular}$\quad$\begin{tabular}{l} 
N/A \\
\hline Keyword:
\end{tabular}


Oxo-analogues of 20-hydroxyecdysone in the synthesis of novel fluorinated ecdysteroid derivatives

Rimma G. Savchenko ", Anastasia V. Apaeva, Svetlana A. Kostyleva, Oleg S. Mozgovoj, Victor N. Odinokov, Lyudmila V. Parfenova

*Corresponding author. Tel.: +7927 2371855

e-mail:ecdysona@gmail.com

Institute of Petrochemistry and Catalysis, Russian Academy of Sciences prosp. Oktyabrya 141, 450075 Ufa, Russian Federation e-mail: ecdysona@gmail.ru 


\begin{abstract}
Novel side chain $\omega$-fluorinated ecdysteroid analogues were obtained starting from $\omega$-functionalized ecdysteroids. Thus, the reaction of diacetonides of $25 R S$ hydroxy- and 24-hydroxy 27-nor- and 25,26,27-tris-nor-20-hydroxyecdysone derivatives with diethylaminosulfur trifluoride (DAST) involves replacement of the terminal hydroxyl group and dehydration at the 14-position to give 25RSfluoro- and 24-fluoro-14-anhydro analogues of ecdysteroids.
\end{abstract}




\section{Introduction}

Ecdysteroids are known as insect hormones which control molting, metamorphosis, and reproduction [1]. Some ecdysteroids act as hormone antagonists, disturbing insect molting process [2]; however, these compounds cannot efficiently affect the population of insects, which has acquired defense mechanisms against exogenous ecdysteroids during evolution. In view of this fact, a synthesis of novel ecdysteroid analogues would provide compounds for efficient regulation of vital processes in insects [3].

The non-hormonal biological activity of ecdysteroids towards mammals is intensively studied [4-6]. It has been reported that non-polar ecdysteroids possess chemo-sensitizing activity towards various cancer cell lines [7-10]. The fluorination of steroids, juvenile hormones, and pheromones can influence the biological activity of these compounds [11]. The interest in fluorinated analogues of natural compounds is caused by features of the fluorine atom, which significantly affects the biological activity and the metabolic stability of a molecule due to high electronegativity, and small size and to low polarizability of the C-F bond. Thus, the introduction of fluorine atom in natural products is the attractive strategy to obtain new leads for drug discovery [12]. The most common synthetic route to fluorinated derivatives is fluorination with various nucleophilic reagents [13]. Among them, diethylaminosulfur trifluoride (DAST) is widely used in deoxyfluorination of oxygen-containing organic compounds; moreover, this reagent is effective for geminal fluorination of acid-sensitive substrates such as aldehydes and ketones [14].

The aim of the present study was to involve new side chain analogues of 20hydroxyecdysone in the DAST-fluorination and to extend the scope of the reaction to the synthesis of biologically active fluorinated analogues of ecdysteroids.

\section{Results and Discussion}


In this regard, we began our research from the study of DAST fluorination of the side chain-modified oxoecdysteroids $\mathbf{1}$ and $\mathbf{2}$, which were obtained by the ozonolysis of $\Delta^{24,25}$ - and $\Delta^{25,26}$-derivatives of 20-hydroxyecdysone [16]. It should be noted that 20-hydroxyecdysone is a polyhydroxylated molecule with three secondary and three tertiary hydroxyl groups and it reacts with the DAST reagent to give a complex reaction mixture. DAST fluorination of ecdysteroids $\mathbf{1}$ and $\mathbf{2}$ having an acid-sensitive $\gamma$-hydroxy- $\alpha, \beta$-enone moiety gave only 14 -anhydro derivatives 3 and $\mathbf{4}$. Moreover, it was found that the oxo groups of ecdysteroid side chain are inert to the DAST-mediated geminal difluorination. That is why terminal alcohols 5 and $\mathbf{6}$ were chosen as alternative precursors for the synthesis of ecdysteroid fluorinated analogues. Such alcohols with the intact native 7-en-6-one chromophore were prepared via chemo- and stereoselective reduction of $\omega$-oxosubstrates 1 and 2 by L-selectride (Scheme 1).

As follows from the integral intensities of the H-25 signal $\left(\delta_{\mathrm{H}} 3.82\right.$ and 4.03 $\mathrm{ppm}$ ) in the ${ }^{1} \mathrm{H}$ NMR spectrum, compound $\mathbf{5}$ is a diastereomeric mixture of $25 S / R$ alcohols present in 85:15 ratio. In order to assign the absolute configuration of the $\mathrm{C}^{25}$ stereogenic center in the major and minor epimers, alcohols 5 were transformed into esters 7 through the reaction with $(R)$-2-methoxy-2-phenylacetyl chloride $(R-\mathrm{MPACl})$. It is known that the major structure of $R$-MPA ester derivatives have the syn-periplanar $(s p)$ conformation, in which $\mathrm{H}-25$ atoms and methoxy and carbonyl groups are located in the same plane [17]. In the conformer of $R$-MPA ester 7, these groups are arranged in such a way that the methyl group $\left(\mathrm{CH}_{3}{ }^{26}\right)$ should be shielded by the phenyl group (Figure 1). The ${ }^{1} \mathrm{H}$ NMR spectrum of a $6: 1$ mixture of diastereomeric esters 7 shows that the signal for the $C^{26}$ methyl group of the major epimer is shifted to the upfield with respect to the minor epimer signals, and the diastereomeric effect is $\Delta \delta^{R S}\left(\mathrm{C}^{26} \mathrm{H}_{3}\right)=0.13 \mathrm{ppm}$. On the contrary, $\mathrm{C}^{24}$-diastereotopic proton signals of the major diastereomer are shifted to the downfield and $\Delta \delta^{R S}\left(\mathrm{C}^{24} \mathrm{H}_{2}\right)=-0.16$ and -0.07 ppm. Taking into account all observed effects, we identify the major diastereomer 7 as $25 S$, whereas the minor one is $25 R$. 
Scheme 1. Reaction of $\omega$-oxoecdysteroids 1, 2 with DAST and L-selectride.
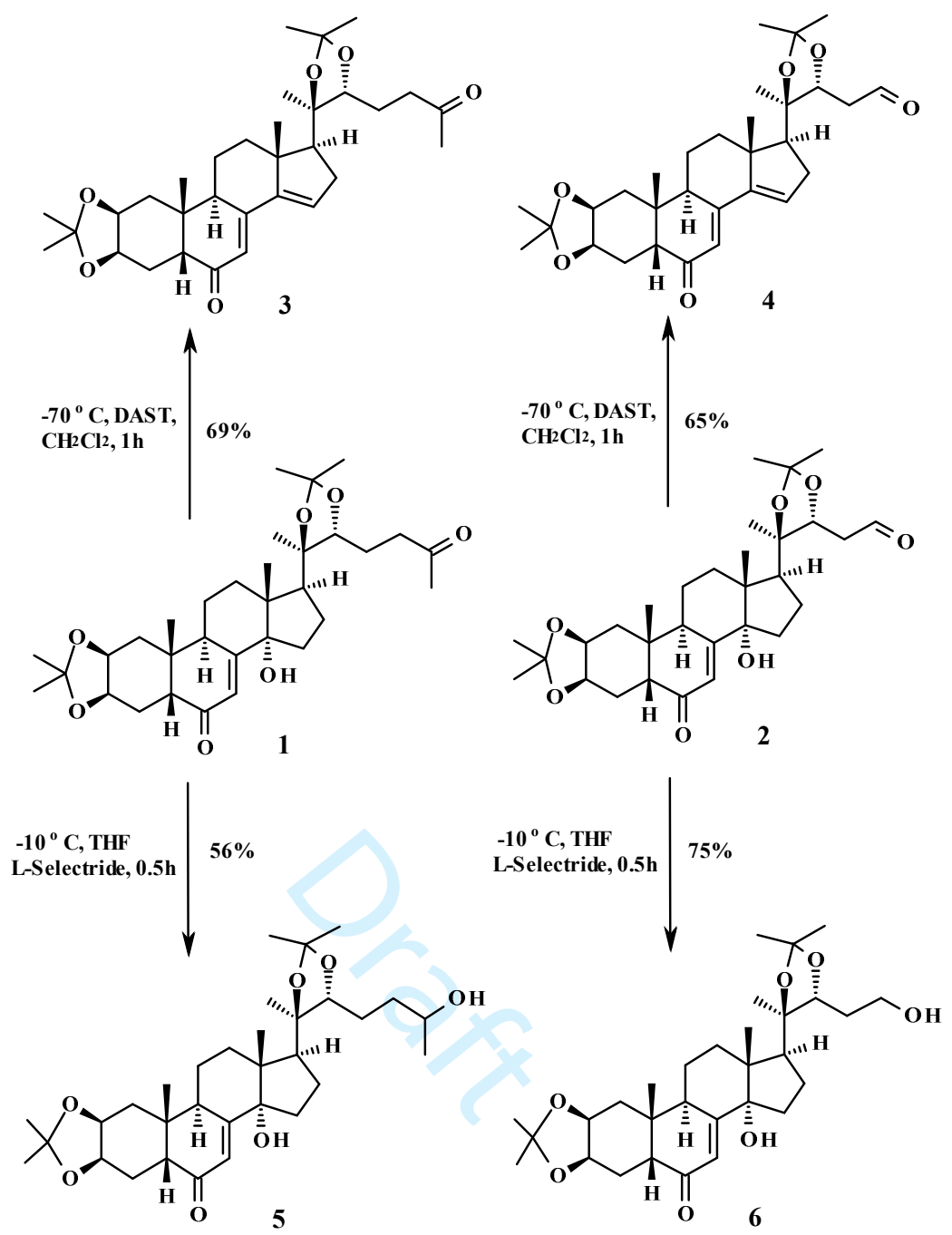

Fig. 1. Model for configurational of the major diastereomer of $R$-MPA ester 7. 


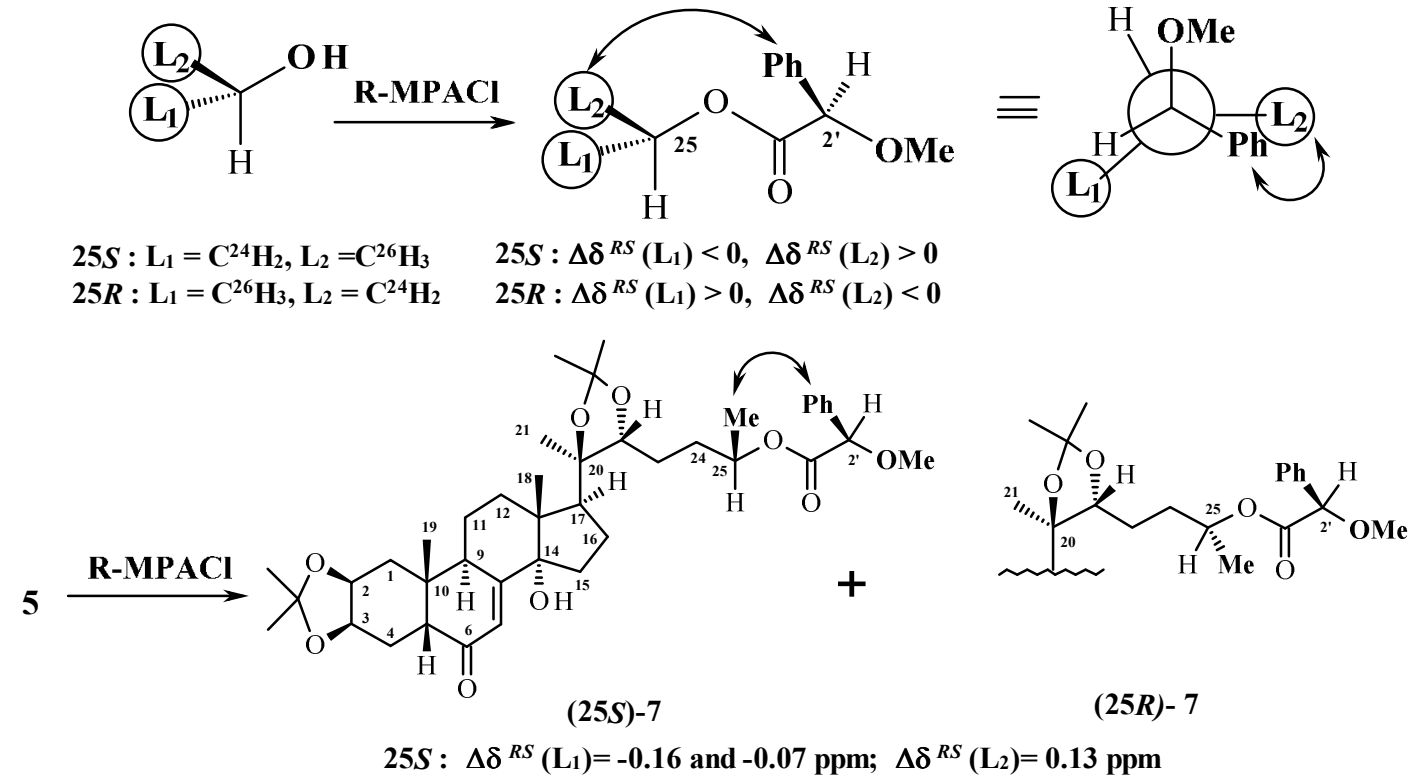

Fluorination of alcohol $\mathbf{5}$ bearing one tertiary and one secondary hydroxyl groups provides a mixture of 14-anhydro-25RS-fluoro-diastereomers $\mathbf{8}$. The formation of the $\Delta^{14,15}$-double bond is confirmed by the presence of two signals at $\delta_{\mathrm{C}} 148.8$ and $128.7 \mathrm{ppm}$ and by the disappearance of the signal for the tertiary C14 atom at $\delta_{\mathrm{C}} 84.85 \mathrm{ppm}$. Previously, the same 14-dehydration product was observed in the fluorination of 20-hydroxyecdysone diacetonide [3]. The substitution of fluorine for the 25-hydroxyl group is evidenced by characteristic downfield shift of the C-25 signal $\left(\Delta \delta_{\mathrm{C}} 21.8 \mathrm{ppm}\right)$ occurring at $91.5 \mathrm{ppm}$ as a doublet $\left(\mathrm{J}_{\mathrm{C}-\mathrm{F}}=162 \mathrm{~Hz}\right)$. The corresponding $\mathrm{H}-25$ signal is revealed as two multiplets with hyperfine structure and equal intensity at $\delta_{\mathrm{H}} 4.64$ and $4.73 \mathrm{ppm}$ (HSQC). The fluorination of secondary and tertiary alcohols catalyzed by DAST follows the $\mathrm{S}_{\mathrm{N}} 1$ mechanism with the involvement of oxonium (A) and carbenium-dication (B) as described previously [18] (Figure 2). The subsequent addition of the fluoride ion to C-25 and the removal of the H-15 proton give diastereomeric mixture 8 .

Fig. 2. Reaction of diol 5 with the DAST-reagent. 


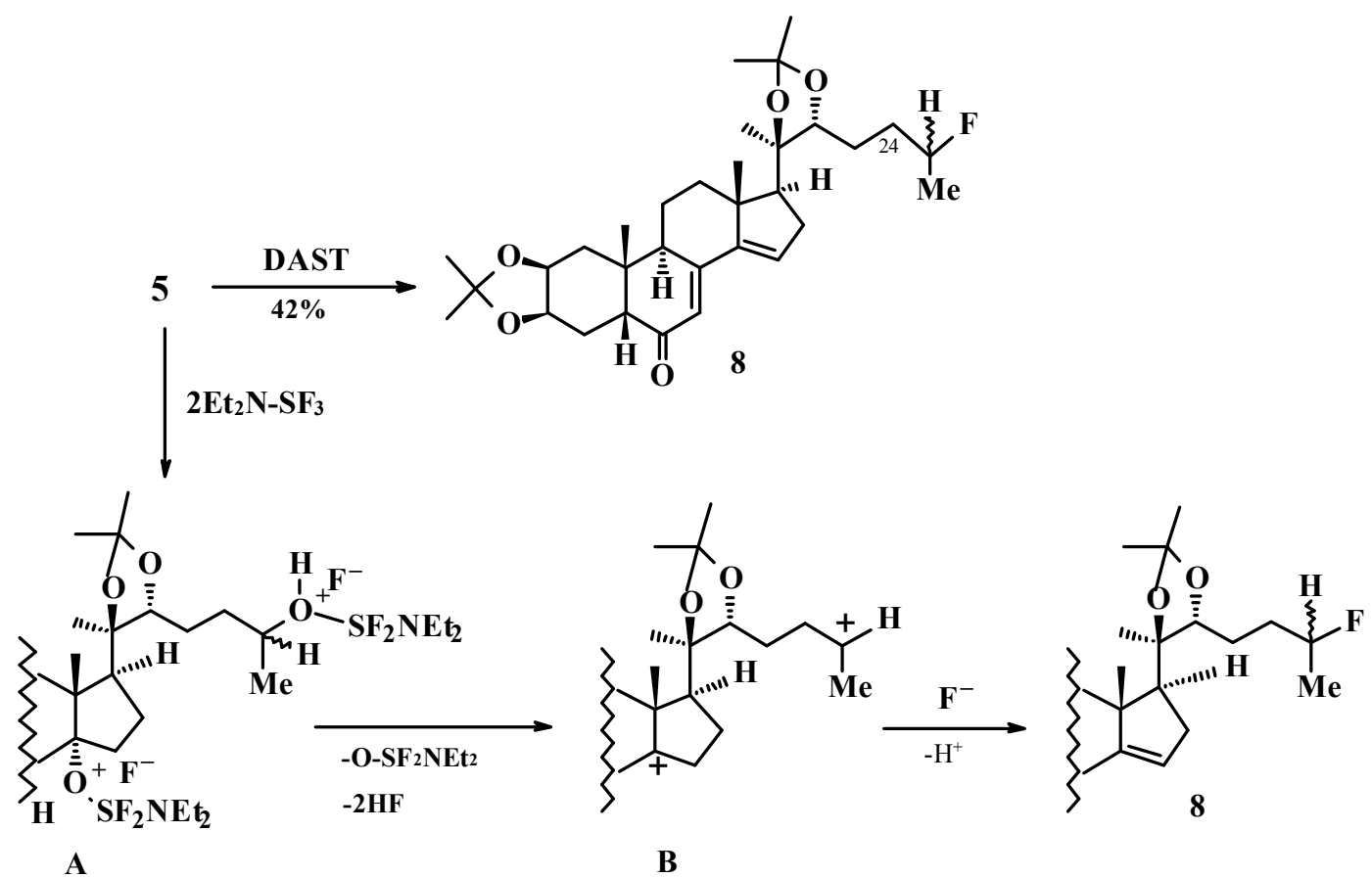

The reaction of primary alcohol 6 with DAST gives isomeric 24-fluoro8(9),14(15)-diene 10 (apparently as a result of allylic rearrangement of H-9 to C-7 atom), in addition to the expected 24-fluoro-7(8),14(15)-diene 9 (similarly to fluorination of alcohol 5). The ${ }^{13} \mathrm{C}$ NMR spectrum of compound 9 exhibits four signals of $s p^{2}$-hybridized carbon atoms, whereas the ${ }^{1} \mathrm{H}$ NMR spectrum contains resonance lines for two olefinic protons at $\delta_{\mathrm{H}} 6.00$ and $6.10 \mathrm{ppm}$, which correlate with the $\mathrm{C}-15$ and $\mathrm{C}-17$ signals in the HSQC experiment, respectively. The assignment of the H-15 signal $\left(\delta_{\mathrm{H}} 6.00 \mathrm{ppm}\right)$ unambiguously follows from the HMBC spectrum, which shows cross-peaks with the C-13 ( $\left.\delta_{\mathrm{C}} 47.49 \mathrm{ppm}\right)$ and C17 signals $\left(\delta_{\mathrm{C}} 57.37 \mathrm{ppm}\right)$. The correlation between the signal at $\mathrm{H}-15$ and the quaternary carbon signal at $\delta_{\mathrm{C}} 148.66 \mathrm{ppm}$ attests that the latter belongs to the C14 atom. The signal of quaternary $s p^{2}$-hybridized carbon atom at $\delta_{\mathrm{C}} 153.67 \mathrm{ppm}$ is assigned to C-8 relying on its correlation with the $\mathrm{H}-7$ signal in the $\mathrm{HMBC}$ spectrum. The ${ }^{13} \mathrm{C}$ NMR spectrum of $\mathbf{1 0}$ also exhibits four signals of $s p^{2}$ hybridized carbon atom; however, unlike the spectrum of compound $\mathbf{9}$, three of the signals correspond to tetrasubstituted atoms (DEPTq) and were assigned to C-8, C9, and C-14 on the basis of HSQC and HMBC data. 
The replacement of the 24-hydroxyl group in compounds $\mathbf{9}$ and $\mathbf{1 0}$ by fluorine atom is evidenced by significant downfield shifts of the C-24 signal $\left(\Delta \delta_{\mathrm{C}} 20.5\right.$ ppm) appearing as a doublet with the coupling constant ${ }^{1} \mathrm{~J}_{C F}=164 \mathrm{~Hz}$. The corresponding ${ }^{1} \mathrm{H}$ NMR signals of the $\mathrm{H}_{2} \mathrm{FC}$-(24)-group are located in lower field and are manifested as hyperfine-structure multiplets $\left(\delta_{\mathrm{H}} 4.60\right.$ and $\left.4.70 \mathrm{ppm}\right)$, which attests to their diastereotopicity.

Fig. 3. Reaction of diol 6 with the DAST-reagent.

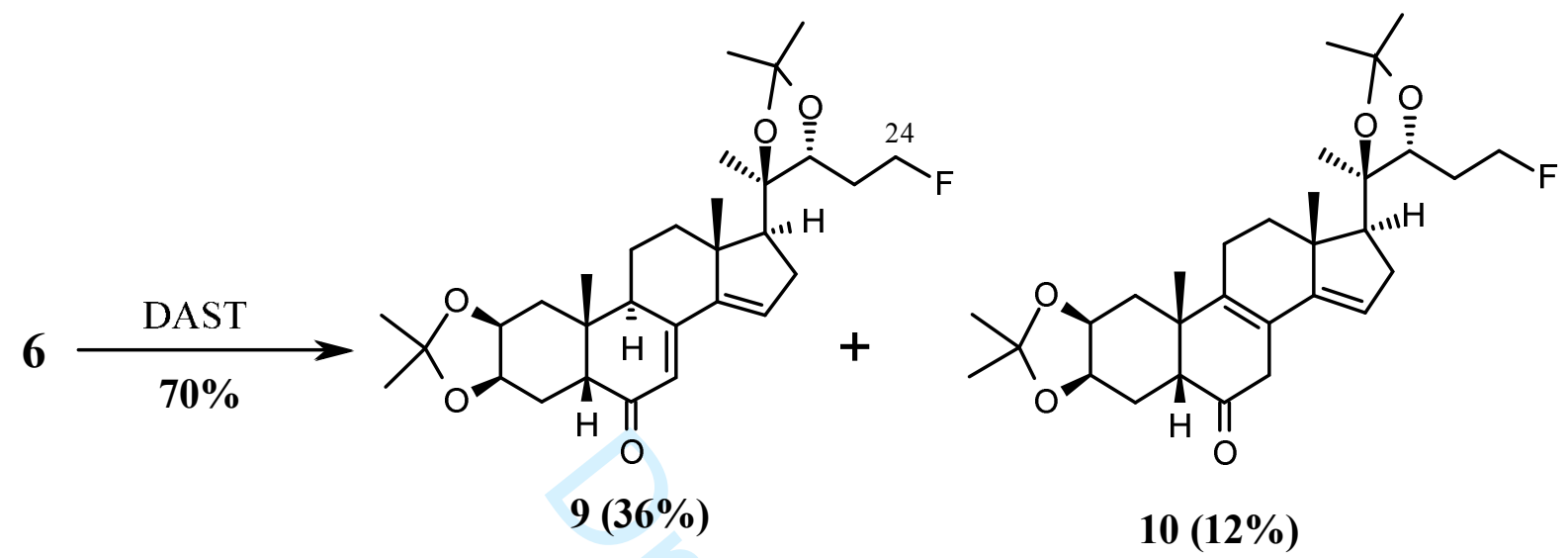

\section{Conclusion}

Thus, novel side chain $\omega$-fluorinated ecdysteroid analogues were obtained starting from $\omega$-functionalized ecdysteroids. It was ascertained that under the action of DAST reagent, dehydration of the tertiary 14-hydroxyl group occurs together with fluorination of side-chain primary or secondary $\omega$-hydroxyls.

\section{Experimental}

One-dimensional $\left({ }^{1} \mathrm{H},{ }^{19} \mathrm{~F},{ }^{13} \mathrm{C}\right)$ and two-dimensional (COSY, NOESY, HSQC, and HMBC) NMR spectra were recorded on a Bruker Avance 500 spectrometer (500.17 MHz for ${ }^{1} \mathrm{H}, 470.65$ for ${ }^{19} \mathrm{~F}$ and $125.78 \mathrm{MHz}$ for ${ }^{13} \mathrm{C}$ ) chemical shifts are reported to $\mathrm{Me}_{4} \mathrm{Si}$ and $\mathrm{CF}_{3} \mathrm{Cl}$. Mass spectra of samples 3, 4 and 8-10 were measured using MALDI TOF/TOF method on a Bruker Autoflex III spectrometer with registration of positive ions. Atmospheric pressure chemical ionization (APCI) mass spectra of samples 5, $\mathbf{6}$ were obtained on a HPLC mass- 
spectrometer LCMS-2010EV (Shimadzu) (direct syringe sample inlet, sample solution was in acetonitrile, mobile phase was acetonitrile/water (95:5)) in positive and negative ions mode at the ionizing electrode potential of $4.5 \mathrm{kV}$ and $-3.5 \mathrm{kV}$, respectively. The mobile phase flow rate was $0.1 \mathrm{~mL} \mathrm{~min}^{-1}$. The temperature of the APCI interface was $250^{\circ} \mathrm{C}$, the heater's and the desolvation line's temperature was 200 and $230{ }^{\circ} \mathrm{C}$, respectively. The nebulizer gas (nitrogen) flow rate was $2.5 \mathrm{~L}$ $\min ^{-1}$. Elemental analysis for compounds 7 was performed on Carlo Erba EA-1108 CHNS- $O$ analyzer.

Specific rotations were measured on Perkin-Elmer-341 polarimeter. Melting points were determined on Boetius hot stage. Column chromatography and TLC were performed using silica gel $(<0.06 \mathrm{~mm})$ and pre-coated silica gel (Silufol plates), respectively; spots were processed by treatment with a solution of 4hydroxy-3-methoxybenzaldehyde in ethanol, acidified with sulfuric acid.

The stock compounds were synthesized from 20-hydroxyecdysone [(mp 239- $240^{\circ} \mathrm{C},[\alpha]_{D}^{20}+54.3^{\circ}$ (c 1.45, MeOH); lit.: mp $246^{\circ} \mathrm{C}$ (EtOAc-MeOH, 9:1), $[\alpha]_{D}^{17}+65.3^{\circ}$ (c 1.0, MeOH) [19]. 20-Hydroxyecdysone was isolated from the juice of Serratula coronata L. [19]. The starting compounds were purchased from Acros Organics.

General procedure for the reaction of $\omega$-oxo-ecdysteroids 1 and 2 with

\section{DAST.}

DAST $(2 \mathrm{mmol})$ was added to the cooled $\left(-70^{\circ} \mathrm{C}\right.$, Ar) and a stirred solution of compound 1 or $2(1 \mathrm{mmol})$ in $5 \mathrm{ml}$ of $\mathrm{CH}_{2} \mathrm{Cl}_{2}$. The reaction mixture was kept under stirring for 1 hour, neutralized with saturated $\mathrm{NaHCO}_{3}$ solution, extracted with $\mathrm{CH}_{2} \mathrm{Cl}_{2}$, the extract was washed with $\mathrm{NaCl}$ solution, dried with $\mathrm{MgSO}_{4}$ and evaporated. After column chromatography, compounds $\mathbf{3}$ and $\mathbf{4}$ were isolated.

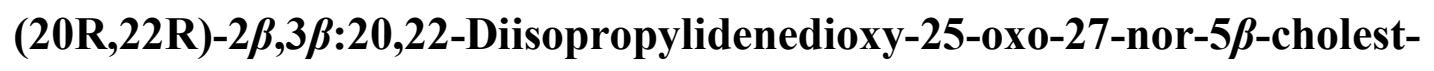

\section{7,14-dien-6-one (3)}

White solid, $0.04 \mathrm{~g}(69 \%), \mathrm{mp} 84-86^{\circ} \mathrm{C},[\alpha]_{D}^{21}-182^{\circ}\left(\mathrm{c} 0.64, \mathrm{CHCl}_{3}\right) .{ }^{1} \mathrm{H}$ NMR (500.17 MHz, $\left.\mathrm{CDCl}_{3}\right) \delta: 0.98$ (s, 3H, 19-Me), 1.06 (s, 3H, 18-Me), 1.16 (s, 
$3 \mathrm{H}, 21-\mathrm{Me}), 1.28$ and $1.95(\mathrm{~m}, 2 \mathrm{H}, 1-\mathrm{H}), 1.29,1.35,1.42$ and $1.50(\mathrm{~s}, 12 \mathrm{H}$, $\left.\underline{\mathrm{Me}}_{2} \mathrm{C}\right), 1.66(\mathrm{~m}, 2 \mathrm{H}, 23-\mathrm{H}), 1.73$ (m, 2H, 11-H), 2.04 (m, 1H, 17-H), 2.18 (s, 3H, 26-Me), 2.23 (m, 2H, 12-H), 2.34(m, 1H, 5-H), 2.36 and 2.62 (m, 2H, 4-H), 2.47 (m, 1H, 9-H), 2.57 and $2.53(\mathrm{~m}, 2 \mathrm{H}, 24-\mathrm{H}), 3.68(\mathrm{~m}, 1 \mathrm{H}, 22-\mathrm{H}), 4.18(\mathrm{~m}, 1 \mathrm{H}, 2-\mathrm{H})$, $4.28(\mathrm{~m}, 1 \mathrm{H}, 3-\mathrm{H}), 5.99$ (d, $1 \mathrm{H}, J=2.5 \mathrm{~Hz}, 7-\mathrm{H}), 6.10(\mathrm{~d}, 1 \mathrm{H}, J=2 \mathrm{~Hz}, 15-\mathrm{H}) .{ }^{13} \mathrm{C}$ NMR $\left(125.78 \mathrm{MHz}, \mathrm{CDCl}_{3}\right) \delta$ : $19.12\left(\mathrm{C}^{18}\right), 20.54\left(\mathrm{C}^{11}\right), 21.17\left(\mathrm{C}^{21}\right), 22.86\left(\mathrm{C}^{23}\right)$, $23.28\left(\mathrm{C}^{19}\right), 26.19,26.87,28.51$ and $28.81\left(2 \underline{\mathrm{Me}}_{2} \mathrm{C}\right), 28.86\left(\mathrm{C}^{26}\right), 30.13\left(\mathrm{C}^{4}\right), 31.54$ $\left(\mathrm{C}^{16}\right), 38.60\left(\mathrm{C}^{9}\right), 38.65\left(\mathrm{C}^{1}\right), 38.40\left(\mathrm{C}^{12}\right), 39.55\left(\mathrm{C}^{10}\right), 40.82\left(\mathrm{C}^{24}\right), 47.48\left(\mathrm{C}^{13}\right)$, $50.76\left(\mathrm{C}^{5}\right), 57.32\left(\mathrm{C}^{17}\right), 71.72\left(\mathrm{C}^{3}\right), 72.08\left(\mathrm{C}^{2}\right), 80.19\left(\mathrm{C}^{22}\right), 83.18\left(\mathrm{C}^{20}\right), 108.23$ and $108.29\left(2 \mathrm{Me}_{2} \underline{\mathrm{C}}\right), 120.69\left(\mathrm{C}^{7}\right), 128.22\left(\mathrm{C}^{15}\right), 148.65\left(\mathrm{C}^{14}\right), 153.87\left(\mathrm{C}^{8}\right), 202.25\left(\mathrm{C}^{6}\right)$, $208.29\left(\mathrm{C}^{25}\right)$. MS (MALDI TOF/TOF), $m / z: 565[\mathrm{M}+\mathrm{K}]^{+}, 549[\mathrm{M}+\mathrm{Na}]^{+}$, calcd for: $\mathrm{C}_{32} \mathrm{H}_{46} \mathrm{O}_{6} 526$.

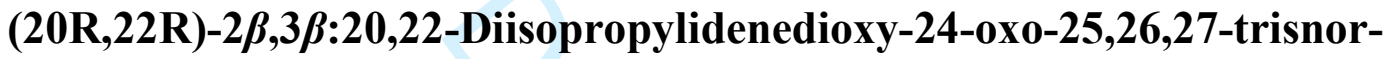
$5 \beta$-cholest-7,14-dien-6-one (4)

White solid, $0.035 \mathrm{~g}(65 \%), \mathrm{mp} 96-98^{\circ} \mathrm{C},[\alpha]_{D}^{21}-287^{\circ}\left(c 1.36, \mathrm{CHCl}_{3}\right) .{ }^{1} \mathrm{H}$ NMR, (500.17 MHz, $\mathrm{CDCl}_{3}$ ) $\delta: 0.99$ (s, 3H, 19-Me), 1.08 (s, 3H, 18-Me), 1.23 (s, $3 \mathrm{H}, 21-\mathrm{Me}), 1.34,1.36,1.45$ and $1.51\left(\mathrm{~s}, 12 \mathrm{H}, \underline{\mathrm{Me}}_{2} \mathrm{C}\right), 1.15-2.73(\mathrm{~m}, 17 \mathrm{H}, \mathrm{CH}$, $\mathrm{CH}_{2}$ ), 4.09-4.30 (m, 3H, 2-H, 3-H, 22-H) 5.99 (d, 1H, $\left.J=2 \mathrm{~Hz}, 7-\mathrm{H}\right), 6.10$ (d, 1H, $J=2.5 \mathrm{~Hz}, 15-\mathrm{H}), 9.83$ (s, 1H, CHO). ${ }^{13} \mathrm{C}$ NMR $\left(125.78 \mathrm{MHz}, \mathrm{CDCl}_{3}\right) \delta: 19.10$ $\left(\mathrm{C}^{18}\right), 20.52\left(\mathrm{C}^{11}\right), 21.50\left(\mathrm{C}^{21}\right), 23.27\left(\mathrm{C}^{19}\right), 26.40,26.83,28.51$ and 28.73 (2.3:20.22 $\left.\underline{\mathrm{Me}}_{2} \mathrm{C}\right), 26.83\left(\mathrm{C}^{4}\right), 31.48\left(\mathrm{C}^{16}\right), 37.58\left(\mathrm{C}^{1}\right), 38.40\left(\mathrm{C}^{12}\right), 39.55\left(\mathrm{C}^{10}\right)$, $39.59\left(\mathrm{C}^{9}\right), 43.39\left(\mathrm{C}^{23}\right), 47.53\left(\mathrm{C}^{13}\right), 50.74\left(\mathrm{C}^{5}\right), 57.34\left(\mathrm{C}^{17}\right), 71.70\left(\mathrm{C}^{3}\right), 72.07$ $\left(\mathrm{C}^{2}\right), 75.28\left(\mathrm{C}^{22}\right), 82.98\left(\mathrm{C}^{20}\right), 108.03$ and $108.33\left(2 \mathrm{Me}_{2} \mathrm{C}\right), 120.83\left(\mathrm{C}^{7}\right), 127.93$ $\left(\mathrm{C}^{15}\right), 148.62\left(\mathrm{C}^{14}\right), 153.83\left(\mathrm{C}^{8}\right), 199.82\left(\mathrm{C}^{24}\right), 202.18\left(\mathrm{C}^{6}\right) . \quad$ MS (MALDI TOF/TOF), $m / z$ : $499[\mathrm{M}+\mathrm{H}]^{+}$, calcd for: $\mathrm{C}_{30} \mathrm{H}_{42} \mathrm{O}_{6} 498$.

\section{General procedure for the reduction of $\omega$-oxo-ecdysteroids 1 and 2.}

A solution of the stirred starting substrate $(0.1 \mathrm{mmol}) \mathbf{1}$ or 2 in $5 \mathrm{ml}$ of dry THF was cooled to $-10{ }^{\circ} \mathrm{C}$ in the argon atmosphere. To the solution 1.2 moles of a $1 \mathrm{M} \mathrm{L}$-Selectride were added. The reaction mixture was stirred for $0.5 \mathrm{~h}$ at $-10^{\circ} \mathrm{C}$, 
and then the temperature was brought to room temperature and stirred for an additional $1 \mathrm{~h}$, monitoring the reaction by TLC until the starting substrate disappeared. At the end of the reaction, the reaction mixture was acidified with saturated $\mathrm{NH}_{4} \mathrm{Cl}$ solution, extracted with EtOAc. The extract was dried with $\mathrm{Na}_{2} \mathrm{SO}_{4}$, evaporated and chromatographed on $\mathrm{SiO}_{2}$, eluent $\mathrm{CHCl}_{3}$.

(20R,22R,25R/S)-14 $\alpha, 25-D i h y d r o x y-2 \beta, 3 \beta$ :20,22-diisopropylidenedioxy27-nor-5 $\boldsymbol{\beta}$-cholest-7-en-6-one (5)

White solid, $0.05 \mathrm{~g}(56 \%), \mathrm{mp} 125-127^{\circ} \mathrm{C},[\alpha]_{D}^{21}+23.8^{\circ}\left(c 0.71, \mathrm{CHCl}_{3}\right) .{ }^{1} \mathrm{H}$ NMR (500.17 MHz, $\left.\mathrm{CDCl}_{3}\right)$ ): 0.78 (s, 3H, 18-H), 0.98 (s, 3H, 19-H), 1.15 (s, 3H, 21-H), 1.20 (d, 3H, $J=6.0 \mathrm{~Hz}, 26-\mathrm{H}), 1.24$ and 1.95 (m, 2H, 1-H), 1.33 and 1.49 (s, 6H, 2,3- $\left.\underline{\mathrm{Me}}_{2} \mathrm{C}\right), 1.34$ and $1.42\left(\mathrm{~s}, 6 \mathrm{H}, 20,22-\mathrm{Me}_{2} \mathrm{C}\right), 1.52$ and $2.02(\mathrm{~m}, 2 \mathrm{H}, 4-\mathrm{H})$, $1.55(\mathrm{~m}, 2 \mathrm{H}, 23-\mathrm{H}), 1.24$ and $1.95(\mathrm{~m}, 2 \mathrm{H}, 24-\mathrm{H}), 1.65$ and $1.72(\mathrm{~m}, 2 \mathrm{H}, 16-\mathrm{H})$, $1.71(\mathrm{~m}, 2 \mathrm{H}, 11-\mathrm{H}), 1.78(\mathrm{~m}, 2 \mathrm{H}, 15-\mathrm{H}), 1.82(\mathrm{~m}, 2 \mathrm{H}, 12-\mathrm{H}), 2.24(\mathrm{~m}, 1 \mathrm{H}, 17-\mathrm{H})$, $2.32(\mathrm{dd}, 1 \mathrm{H}, J=5.0 \mathrm{~Hz}$ and $J=12.0 \mathrm{~Hz}, 5-\mathrm{H}), 2.81(\mathrm{~m}, 1 \mathrm{H}, 9-\mathrm{H}), 3.82(\mathrm{~m}, 1 \mathrm{H}$, minor diastereomer, $25 R, 25-\mathrm{H}), 4.03(\mathrm{~m}, 1 \mathrm{H}$, major diastereomer, $25 S, 25-\mathrm{H})$, $3.70(\mathrm{~m}, 1 \mathrm{H}, 22-\mathrm{H}), 4.24(\mathrm{~m}, 1 \mathrm{H}, 2-\mathrm{H}), 4.27(\mathrm{~m}, 1 \mathrm{H}, 3-\mathrm{H}), 5.81(\mathrm{~s}, 1 \mathrm{H}, 7-\mathrm{H}) .{ }^{13} \mathrm{C}$ NMR (125.78 MHz, $\left.\mathrm{CDCl}_{3}\right) \delta: 17.02\left(\mathrm{C}^{18}\right), 20.52\left(\mathrm{C}^{11}\right), 21.19\left(\mathrm{C}^{16}\right), 21.78\left(\mathrm{C}^{21}\right)$, $23.59\left(\mathrm{C}^{19}\right.$ and $\left.\mathrm{C}^{26}\right), 25.95\left(\mathrm{C}^{23}\right), 26.46$ and $26.87\left(20,22 \underline{\mathrm{Me}}_{2} \mathrm{C}\right), 26.66\left(\mathrm{C}^{15}\right), 28.54$ and $28.91\left(2,3 \underline{\mathrm{Me}}_{2} \mathrm{C}\right), 30.97\left(\mathrm{C}^{12}\right), 31.59\left(\mathrm{C}^{4}\right), 34.50\left(\mathrm{C}^{9}\right), 37.41\left(\mathrm{C}^{1}\right), 37.58\left(\mathrm{C}^{10}\right)$, $37.78\left(\mathrm{C}^{24}\right), 47.45\left(\mathrm{C}^{13}\right), 48.99\left(\mathrm{C}^{17}\right), 50.79\left(\mathrm{C}^{5}\right), 68.14\left(\mathrm{C}^{25}\right), 71.62\left(\mathrm{C}^{3}\right), 72.14$ $\left(\mathrm{C}^{2}\right), 81.71\left(\mathrm{C}^{22}\right), 84.50\left(\mathrm{C}^{20}\right), 84.85\left(\mathrm{C}^{14}\right), 107.67$ and $108.29\left(2 \mathrm{Me}_{2} \mathrm{C}\right), 121.32$ $\left(\mathrm{C}^{7}\right), 163.42\left(\mathrm{C}^{8}\right), 202.92\left(\mathrm{C}^{6}\right)$. MS (APCI), m/z: $547[M+\mathrm{H}]^{+}, 581[M+\mathrm{Cl}]^{-}$, calcd for: $\mathrm{C}_{32} \mathrm{H}_{50} \mathrm{O}_{7} 546$.

\section{(20R,22R)-14 $\alpha, 24-D i h y d r o x y-2 \beta, 3 \beta: 20,22$-diisopropylidenedioxy-}

\section{5,26,27-trisnor-5 $\beta$-cholest-7-en-6-one (6)}

White solid, $0.063 \mathrm{~g}(75 \%), \mathrm{mp} 138-140^{\circ} \mathrm{C},[\alpha]_{D}^{21}+28.1^{\circ}\left(c 0.75, \mathrm{CHCl}_{3}\right) .{ }^{1} \mathrm{H}$ NMR (500.17 MHz, $\mathrm{CDCl}_{3}$ ) $\delta: 0.76$ (s, 3H, 18-Me), 0.95 (s, 3H, 19-Me), 1.19 (s, $3 \mathrm{H}, 21-\mathrm{Me}), 1.20$ and $1.94(\mathrm{~m}, 2 \mathrm{H}, 1-\mathrm{H}), 1.30 ; 1.31 ; 1.39$ and $1.46(\mathrm{~s}, 12 \mathrm{H}$, 2,3:20,22- $\left.\mathrm{Me}_{2} \mathrm{C}\right), 1.74$ and $1.98(\mathrm{~m}, 2 \mathrm{H}, 4-\mathrm{H}), 1.80$ and $2.20(\mathrm{~m}, 2 \mathrm{H}, 15-\mathrm{H}), 1.82$ and $1.98(\mathrm{~m}, 2 \mathrm{H}, 16-\mathrm{H}), 2.23(\mathrm{~m}, 1 \mathrm{H}, 17-\mathrm{H}), 2.38(\mathrm{dd}, 1 \mathrm{H}, J=5.0 \mathrm{~Hz}$ and $J=12.5$ 
Hz, 5-H), 2.83 (m, 1H, 9-H), $3.86(\mathrm{dd}, 1 \mathrm{H}, J=1.5 \mathrm{~Hz}$ and $J=10.5 \mathrm{~Hz}, 22-\mathrm{H}), 4.20$ $\mathrm{m}(1 \mathrm{H}, 2-\mathrm{H}), 4.23 \mathrm{~m}(1 \mathrm{H}, 3-\mathrm{H}), 5.78 \mathrm{~s}(1 \mathrm{H}, 7-\mathrm{H}) .{ }^{13} \mathrm{C} \mathrm{NMR}\left(125.78 \mathrm{MHz}, \mathrm{CDCl}_{3}\right)$

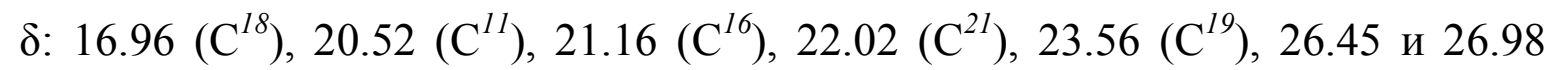
(20,22 $\left.\underline{\mathrm{Me}}_{2} \mathrm{C}\right), 26.59\left(\mathrm{C}^{15}\right), 28.52$ and $28.93\left(2,3 \underline{\mathrm{Me}}_{2} \mathrm{C}\right), 30.95\left(\mathrm{C}^{12}\right), 31.31\left(\mathrm{C}^{4}\right.$ and $\left.\mathrm{C}^{23}\right), 34.48\left(\mathrm{C}^{9}\right), 37.53\left(\mathrm{C}^{1}\right), 37.74\left(\mathrm{C}^{10}\right), 47.43\left(\mathrm{C}^{13}\right), 48.95\left(\mathrm{C}^{17}\right), 50.76\left(\mathrm{C}^{5}\right)$, $61.22\left(\mathrm{C}^{24}\right), 71.62\left(\mathrm{C}^{3}\right), 72.12\left(\mathrm{C}^{2}\right), 79.79\left(\mathrm{C}^{22}\right), 84.34\left(\mathrm{C}^{20}\right), 84.63\left(\mathrm{C}^{14}\right), 107.54$ and $108.27\left(2 \mathrm{Me}_{2} \underline{\mathrm{C}}\right), 121.15\left(\mathrm{C}^{7}\right), 163.90\left(\mathrm{C}^{8}\right), 203.17\left(\mathrm{C}^{6}\right) . \mathrm{MS}(\mathrm{APCI}), \mathrm{m} / z: 519$ $[M+\mathrm{H}]^{+}, 553[M+\mathrm{Cl}]^{-}$, calcd for: $\mathrm{C}_{30} \mathrm{H}_{46} \mathrm{O}_{7} 518$.

R-MPA ester of $(20 R, 22 R, 25 R / S)-14 \alpha, 25-D i h y d r o x y-2 \beta, 3 \beta: 20,22-$ diisopropylidenedioxy-27-nor-5 $\boldsymbol{\beta}$-cholest-7-en-6-one (7).

To a solution of alcohol $50.016 \mathrm{~g}(0.03 \mathrm{mmol})$ in $2 \mathrm{ml}$ of pyridine the $R$ MPA anhydride chloride $0.01 \mathrm{ml}(0.05 \mathrm{mmol})$ and $0.003 \mathrm{~g}$ of DMAP were added successively,. The reaction mixture was stirred for 8 hours, evaporated and chromatographed $\left(\mathrm{SiO}_{2}\right.$, eluent $\left.\mathrm{CHCl}_{3}\right)$.

White solid, 0.012g (78\%), mp 138-140 ${ }^{\circ} \mathrm{C} .{ }^{1} \mathrm{H}$ NMR (500.17 MHz, $\mathrm{CDCl}_{3}$ ) $\delta: 0.79$ (s, 3H, 18-Me), 1.01 (s, 3H, 19-Me), 1.07 (s, 3H, 21-Me), 1.15 (d, 3H, $J=$ $6.0 \mathrm{~Hz}$, major diastereomer 25S, 26-Me), 1.27 and $1.98(\mathrm{~m}, 2 \mathrm{H}, 1-\mathrm{H}), 1.28(\mathrm{~m}, 3 \mathrm{H}$, minor diastereomer, 25R, 26-Me), 1.32 and 1.42 (s, $6 \mathrm{H}, 2,3-\mathrm{Me}_{2} \mathrm{C}$ ), 1.36 and 1.52 \left. (s, 6H, 20,22- ${\underline{-\mathrm{Me}_{2}}}_{2} \mathrm{C}\right), 1.45(\mathrm{~m}, 2 \mathrm{H}, 23-\mathrm{H}), 1.55(\mathrm{~m}, 2 \mathrm{H}, 15-\mathrm{H}), 1.52$ and $1.71(\mathrm{~m}$, $2 \mathrm{H}$, minor diastereomer, 25R, 24-H), 1.59 and $1.87(\mathrm{~m}, 2 \mathrm{H}$, major diastereomer 25S, 24-H), 1.66 and $1.75(\mathrm{~m}, 2 \mathrm{H}, 16-\mathrm{H}), 1.82(\mathrm{~m}, 2 \mathrm{H}, 11-\mathrm{H}), 1.85$ and $2.15(\mathrm{~m}$, 2H, 4-H), $1.86(\mathrm{~m}, 2 \mathrm{H}, 12-\mathrm{H}), 2.11(\mathrm{~m}, 1 \mathrm{H}, 17-\mathrm{H}), 2.38(\mathrm{~m}, 1 \mathrm{H}, 5-\mathrm{H}), 2.83(\mathrm{~m}$, $1 \mathrm{H}, 9-\mathrm{H}), 3.44$ (s, $\left.3 \mathrm{H}, \mathrm{OCH}_{3}\right), 3.64$ (d, $\left.1 \mathrm{H}, J=9.5 \mathrm{~Hz}, 22-\mathrm{H}\right), 4.26(\mathrm{~m}, 1 \mathrm{H}, 3-\mathrm{H})$, $4.30(\mathrm{~m}, 1 \mathrm{H}, 2-\mathrm{H}), 4.78\left(\mathrm{~s}, 1 \mathrm{H}, 2^{\prime}-\mathrm{H}\right), 4.95(\mathrm{~m}, 1 \mathrm{H}$, minor diastereomer 25R, 25$\mathrm{H}), 5.08(\mathrm{~m}, 1 \mathrm{H}$, major diastereomer $25 S, 25-\mathrm{H}), 5.85(\mathrm{~s}, 1 \mathrm{H}, 7-\mathrm{H}), 7.34-7.46(\mathrm{~m}$, 5H, Ph). ${ }^{13} \mathrm{C}$ NMR (125.78 MHz, $\left.\mathrm{CDCl}_{3}\right) \delta: 17.01\left(\mathrm{C}^{18}\right), 19.38\left(\mathrm{C}^{26}\right), 20.58\left(\mathrm{C}^{16}\right)$, 21.05( $\left.\mathrm{C}^{11}\right), 21.80\left(\mathrm{C}^{21}\right), 23.64\left(\mathrm{C}^{19}\right), 25.95\left(\mathrm{C}^{23}\right), 26.47$ and $26.89\left(20,22 \mathrm{Me}_{2} \mathrm{C}\right)$, $26.70\left(\mathrm{C}^{4}\right.$ and $\left.\mathrm{C}^{15}\right), 28.55$ and $28.99\left(2,3 \underline{\mathrm{Me}_{2}} \mathrm{C}\right), 31.03\left(\mathrm{C}^{12}\right), 33.51\left(\mathrm{C}^{24}\right), 34.63$ $\left(\mathrm{C}^{9}\right), 37.61\left(\mathrm{C}^{1}\right), 37.77\left(\mathrm{C}^{10}\right), 47.51\left(\mathrm{C}^{13}\right), 48.97\left(\mathrm{C}^{17}\right), 50.82\left(\mathrm{C}^{5}\right), 57.27\left(\mathrm{OCH}_{3}\right)$, $71.33\left(\mathrm{C}^{25}\right.$, major diastereomer $\left.25 S\right), 71.63\left(\mathrm{C}^{3}\right), 72.20\left(\mathrm{C}^{2}\right), 72.31\left(\mathrm{C}^{25}\right.$, minor 
diastereomer $25 R), 80.58\left(\mathrm{C}^{22}\right), 84.07\left(\mathrm{C}^{20}\right), 85.03\left(\mathrm{C}^{14}\right), 107.05$ and 108.30 $\left(2 \mathrm{Me}_{2} \underline{\mathrm{C}}\right), 121.43\left(\mathrm{C}^{7}\right), 127.13,127.43,128.26,136.27(\mathrm{Ph}) .163 .42\left(\mathrm{C}^{8}\right), 170.00$ $\left(\mathrm{C}^{1^{\prime}}\right), 202.73\left(\mathrm{C}^{6}\right)$. Anal. Calcd for $\mathrm{C}_{41} \mathrm{H}_{58} \mathrm{O}_{9}$ (694.89): C, 70.87; H, 8.41. Found: $\mathrm{C}$, $70.81 ; \mathrm{H}, 8.38$.

\section{General procedure for the deoxyfluorination of alcohols 5 and 6.}

To the cooled $\left(-70^{\circ} \mathrm{C}, \mathrm{Ar}\right)$ and a stirred solution of compound 5 or $6(1 \mathrm{mmol})$ in 5 $\mathrm{ml}$ of $\mathrm{CH}_{2} \mathrm{Cl}_{2}$ DAST $(1.2 \mathrm{mmol})$ was added. The reaction mixture was kept under stirring for 1 hour, neutralized with saturated $\mathrm{NaHCO}_{3}$ solution, extracted with $\mathrm{CH}_{2} \mathrm{Cl}_{2}$, the extract was washed with $\mathrm{NaCl}$ solution, dried with $\mathrm{MgSO}_{4}$ and evaporated. After column chromatography (eluent, compounds 8, 9 and 10 were isolated.

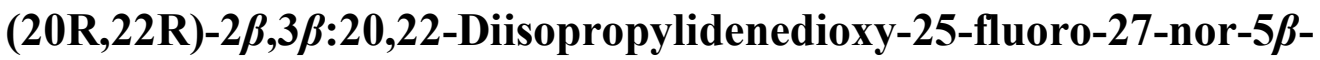

\section{cholest-7,14-dien-6-one (8)}

White solid, $0.012 \mathrm{~g}\left(42 \%\right.$.), mp $100-102^{\circ} \mathrm{C},[\alpha]_{D}^{21}-16^{\circ}\left(c 0.82, \mathrm{CHCl}_{3}\right) .{ }^{1} \mathrm{H}$ NMR (500.17 MHz, $\mathrm{CDCl}_{3}$ ) $\delta: 0.99$ (s, 3H, 19-Me), 1.08 (s, 3H, 18-Me), 1.21 (s, $3 \mathrm{H}, 21-\mathrm{Me}), 1.27$ and $1.97(\mathrm{~m}, 2 \mathrm{H}, 1-\mathrm{H}), 1.32 ; 1.35 ; 1.44$ and $1.51(\mathrm{~s}, 12 \mathrm{H}$, 2,3:20,22 $\mathrm{Me}_{2} \mathrm{C}$ ), 1.32 and $1.76(\mathrm{~m}, 2 \mathrm{H}, 4-\mathrm{H}), 1.39$ (d, 3H, $\left.J=6.0 \mathrm{~Hz}, 26-\mathrm{Me}\right)$, 1.48 and $2.22(\mathrm{~m}, 2 \mathrm{H}, 12-\mathrm{H}), 1.58$ and $1.62(\mathrm{~m}, 2 \mathrm{H}, 23-\mathrm{H}), 1.64(\mathrm{~m}, 2 \mathrm{H}, 24-\mathrm{H})$, $1.76(\mathrm{~m}, 2 \mathrm{H}, 11-\mathrm{H}), 1.99(\mathrm{~m}, 1 \mathrm{H}, 17-\mathrm{H}), 2.36(\mathrm{~d}, 1 \mathrm{H}, J=5.0 \mathrm{~Hz}, 5-\mathrm{H}), 2.63(\mathrm{~m}$, 2H, 16-H), 2.66 (m, 1H, 9-H), 3.73 (dd, 1H, $J=3 \mathrm{~Hz} ; J=10 \mathrm{~Hz}, 22-\mathrm{H}), 4.18$ (m, 1H, 3-H), 4.28 (m, 1H, 2-H), 4.64 and 4.73 (m, 1H, 25-H), 5.99 (s, 1H, 15-H), 6.10 $(\mathrm{d}, 1 \mathrm{H}, J=2.0 \mathrm{~Hz}, 7-\mathrm{H}) \cdot{ }^{13} \mathrm{C}$ NMR $\left(125.78 \mathrm{MHz}, \mathrm{CDCl}_{3}\right) \delta: 19.10\left(\mathrm{C}^{18}\right), 20.55$ $\left(\mathrm{C}^{11}\right), 21.21\left(\mathrm{C}^{21}\right), 21.29\left(\mathrm{C}^{26},{ }^{2} J_{\mathrm{C}-\mathrm{F}}=25.0 \mathrm{~Hz}\right), 23.28\left(\mathrm{C}^{19}\right), 25.27\left(\mathrm{C}^{23}\right), 26.41$ and $26.83\left(20,22 \underline{\mathrm{Me}_{2} \mathrm{C}}\right), 26.83\left(\mathrm{C}^{4}\right), 28.52$ and $28.90\left(2,3 \underline{\mathrm{Me}_{2} \mathrm{C}}\right), 31.59\left(\mathrm{C}^{16}\right), 35.09$ $\left(\mathrm{C}^{24}, J_{C-F}=20.6 \mathrm{~Hz}\right), 37.60\left(\mathrm{C}^{1}\right), 38.41\left(\mathrm{C}^{9}\right), 38.66\left(\mathrm{C}^{10}\right), 39.58\left(\mathrm{C}^{12}\right), 47.51\left(\mathrm{C}^{13}\right)$, $50.76\left(\mathrm{C}^{5}\right), 57.50\left(\mathrm{C}^{17}\right), 71.73\left(\mathrm{C}^{3}\right), 72.09\left(\mathrm{C}^{2}\right), 81.17\left(\mathrm{C}^{22}\right), 83.12\left(\mathrm{C}^{20}\right), 91.51 \mathrm{~d}$ $\left(\mathrm{C}^{25}, J_{\mathrm{C}-\mathrm{F}}=162.0 \mathrm{~Hz}\right), 107.08$ and $108.31\left(2 \mathrm{Me}_{2} \underline{\mathrm{C}}\right), 120.73\left(\mathrm{C}^{7}\right), 128.73\left(\mathrm{C}^{15}\right)$, $148.76\left(\mathrm{C}^{14}\right), 153.75\left(\mathrm{C}^{8}\right), 202.22\left(\mathrm{C}^{6}\right) .{ }^{19} \mathrm{~F}$ NMR $\left(\mathrm{CDCl}_{3}, \mathrm{CFCl}_{3}, \mathrm{MHz}\right) \delta 85.80$ $\left(\mathrm{C}^{25} \mathrm{HF}\right.$ ). MS (MALDI TOF/TOF), $m / z 531[M+\mathrm{H}]^{+}$, calcd for: $\mathrm{C}_{32} \mathrm{H}_{47} \mathrm{FO}_{5} 530$. 
14

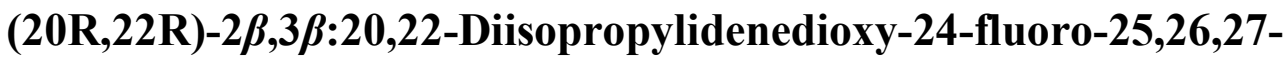

trisnor-5 $\beta$-cholest-7,14-dien-6-one (9)

White solid, 0.015g (36\%), mp 90-92 ${ }^{\circ} \mathrm{C},[\alpha]_{D}^{21}-58.1^{\circ}\left(c \quad 0.16, \mathrm{CHCl}_{3}\right) .{ }^{1} \mathrm{H}$ NMR (500.17 MHz, $\mathrm{CDCl}_{3}$ ) $\delta: 0.99$ (s, 3H, 19-Me), 1.08 (s, 3H, 18-Me), 1.22 (s, $3 \mathrm{H}, 21-\mathrm{Me}), 1.28$ and $1.96(\mathrm{~m}, 2 \mathrm{H}, 1-\mathrm{H}), 1.34 ; 1.35 ; 1.45$ and $1.51(\mathrm{~s}, 12 \mathrm{H}$, 2.3:20.22 $\left.\mathrm{Me}_{2} \mathrm{C}\right), 1.44$ and $2.22(\mathrm{~m}, 2 \mathrm{H}, 12-\mathrm{H}), 1.76(\mathrm{~m}, 2 \mathrm{H}, 11-\mathrm{H}), 1.78(\mathrm{~m}, 2 \mathrm{H}$, 4-H), 1.81 and $1.86(\mathrm{~m}, 2 \mathrm{H}, 23-\mathrm{H}), 2.00(\mathrm{dd}, 1 \mathrm{H}, J=8.0 \mathrm{~Hz} ; 11.0 \mathrm{~Hz}, 17-\mathrm{H}), 2.34$ $(\mathrm{dd}, 1 \mathrm{H}, J=5.0 \mathrm{~Hz} ; 13.0 \mathrm{~Hz}, 5-\mathrm{H}), 2.36(\mathrm{~m}, 2 \mathrm{H}, 9-\mathrm{H}), 2.67$ (dd, $1 \mathrm{H}, J_{16,16}=15.5$ $\left.\mathrm{Hz} ; J_{16,17}=11.0 \mathrm{~Hz}, 16-\mathrm{H}\right), 3.95(\mathrm{~m}, 1 \mathrm{H}, 22-\mathrm{H}), 4.17(\mathrm{~m}, 1 \mathrm{H}, 2-\mathrm{H}), 4.28(\mathrm{~m}, 1 \mathrm{H}, 3-$ $\mathrm{H}), 4.56$ and $4.65(\mathrm{~m}, 2 \mathrm{H}, 24-\mathrm{H}), 6.00(\mathrm{t}, 1 \mathrm{H}, J=2.0 \mathrm{~Hz}, 15-\mathrm{H}), 6.10(\mathrm{~d}, 1 \mathrm{H}, J=$ $2.0 \mathrm{~Hz}, 7-\mathrm{H}) .{ }^{13} \mathrm{C}$ NMR $\left(125.78 \mathrm{MHz}, \mathrm{CDCl}_{3}\right) \delta: 19.12\left(\mathrm{C}^{18}\right), 20.55\left(\mathrm{C}^{11}\right), 21.18$ $\left(\mathrm{C}^{21}\right), 23.28\left(\mathrm{C}^{19}\right), 26.41$ and $26.82\left(20,22 \underline{\mathrm{Me}}_{2} \mathrm{C}\right), 26.83\left(\mathrm{C}^{4}\right), 28.52$ and $28.84(2,3$ $\left.\underline{\mathrm{Me}_{2}} \mathrm{C}\right), 30.35\left(\mathrm{C}^{23}, J=19.5 \mathrm{~Hz}\right), 31.55\left(\mathrm{C}^{16}\right), 37.60\left(\mathrm{C}^{1}\right), 38.64\left(\mathrm{C}^{10}\right), 38.84\left(\mathrm{C}^{9}\right)$, $39.61\left(\mathrm{C}^{12}\right), 47.49\left(\mathrm{C}^{13}\right), 50.76\left(\mathrm{C}^{5}\right), 57.37\left(\mathrm{C}^{17}\right), 71.72\left(\mathrm{C}^{3}\right), 72.09\left(\mathrm{C}^{2}\right), 76.77$ $\left(\mathrm{C}^{22}\right), 81.62\left(\mathrm{C}^{24}, J_{C F}=165.0 \mathrm{~Hz}\right), 82.95\left(\mathrm{C}^{20}\right), 107.45$ and $108.32\left(2 \mathrm{Me}_{2} \mathrm{C}\right), 120.76$ $\left(\mathrm{C}^{7}\right), 128.13\left(\mathrm{C}^{15}\right), 148.66\left(\mathrm{C}^{14}\right), 153.67\left(\mathrm{C}^{8}\right), 202.19\left(\mathrm{C}^{6}\right) .{ }^{19} \mathrm{~F}$ NMR $\left(\mathrm{CDCl}_{3}\right.$, $\left.\mathrm{CFCl}_{3}, \mathrm{MHz}\right) \delta: 85.81\left(\mathrm{C}^{24} \mathrm{H}_{2} \mathrm{~F}\right)$. MS (MALDI TOF/TOF), $m / z 503[M+\mathrm{H}]^{+}, 525$ $[M+\mathrm{Na}]^{+}, 541[M+\mathrm{K}]^{+}$, calcd for: $\mathrm{C}_{30} \mathrm{H}_{43} \mathrm{FO}_{5} 502$.

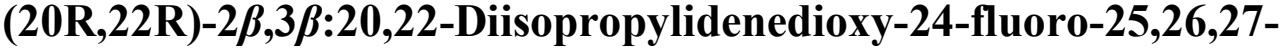

\section{trisnor-5 $\beta$-cholest-8,14-dien-6-one (10)}

White solid, $0.005 \mathrm{~g}(12 \%), \mathrm{mp} 50-52^{\circ} \mathrm{C},[\alpha]_{D}^{21}-9.6^{\circ}\left(c 0.06, \mathrm{CHCl}_{3}\right)$. NMR ${ }^{1} \mathrm{H}\left(500.17 \mathrm{MHz}, \mathrm{CDCl}_{3}\right)$ \&: 1.17 (s, 3H, 19-Me), 1.02 (s, 3H, 18-Me), 1.23 (s, 3H, $21-\mathrm{Me}), 1.28 ; 1.35 ; 1.45$ and $1.53\left(\mathrm{~s}, 12 \mathrm{H}, 2,3: 20,22 \underline{\mathrm{Me}}_{2} \mathrm{C}\right), 1.50$ and $2.20(\mathrm{~m}, 2 \mathrm{H}$, 12-H), 1.60 and 1.98 (m, 2H, 1-H), 1.75 (m, 2H, 23-H), 1.87 (dd, 1H, $J=7.6 \mathrm{~Hz}$; $10.8 \mathrm{~Hz}, 17-\mathrm{H}), 1.98$ and $2.07(\mathrm{~m}, 2 \mathrm{H}, 4-\mathrm{H}), 2.16(\mathrm{~m}, 2 \mathrm{H}, 11-\mathrm{H}), 2.35$ and $2.71(\mathrm{~m}$, 2H, 16-H), 2.55 (dd, 1H, $J=4.8 \mathrm{~Hz} ; 10.0 \mathrm{~Hz}, 5-\mathrm{H}), 2.83$ (d, $2 \mathrm{H},{ }^{2} J_{7,7}=21.0 \mathrm{~Hz}, 7-$ H), $3.97(\mathrm{~m}, 1 \mathrm{H}, 22-\mathrm{H}), 4.10(\mathrm{~m}, 1 \mathrm{H}, 2-\mathrm{H}), 4.30(\mathrm{~m}, 1 \mathrm{H}, 3-\mathrm{H}), 4.55$ and $4.66(\mathrm{~m}$, $2 \mathrm{H}, 24-\mathrm{H}), 5.41$ (s, 1H, 15-H). NMR ${ }^{13} \mathrm{C}\left(125.78 \mathrm{MHz}, \mathrm{CDCl}_{3}\right) \delta: 17.43\left(\mathrm{C}^{18}\right)$, $21.24\left(\mathrm{C}^{21}\right), 23.36\left(\mathrm{C}^{11}\right), 26.43\left(\mathrm{C}^{4}\right), 28.39\left(\mathrm{C}^{19}\right), 26.87$ and $28.49\left(20,22 \underline{\mathrm{Me}}_{2} \mathrm{C}\right)$, 28.89 and $29.71\left(2,3 \underline{\mathrm{Me}}_{2} \mathrm{C}\right), 30.39\left(\mathrm{C}^{23}, J=19.0 \mathrm{~Hz}\right), 31.50\left(\mathrm{C}^{16}\right), 36.57\left(\mathrm{C}^{12}\right)$, 
$37.39\left(\mathrm{C}^{1}\right), 38.85\left(\mathrm{C}^{7}\right), 42.12\left(\mathrm{C}^{10}\right), 45.90\left(\mathrm{C}^{13}\right), 53.13\left(\mathrm{C}^{5}\right), 55.96\left(\mathrm{C}^{17}\right), 71.26\left(\mathrm{C}^{3}\right)$, $72.80\left(\mathrm{C}^{2}\right), 76.70\left(\mathrm{C}^{22}\right), 81.67\left(\mathrm{C}^{24}, J_{C-F}=164.0 \mathrm{~Hz}\right), 83.13\left(\mathrm{C}^{20}\right), 121.73\left(\mathrm{C}^{8}\right)$, 107.35 and $108.40\left(2 \mathrm{Me}_{2} \mathrm{C}\right), 119.15\left(\mathrm{C}^{15}\right), 136.36\left(\mathrm{C}^{9}\right), 147.91\left(\mathrm{C}^{14}\right), 211.53\left(\mathrm{C}^{6}\right)$. ${ }^{19} \mathrm{~F} \mathrm{NMR}\left(\mathrm{CDCl}_{3}, \mathrm{CFCl}_{3}, \mathrm{MHz}\right) \delta: 85.79$. MS (MALDI TOF/TOF), m/z 503 $[M+\mathrm{H}]^{+}$, calcd for $\mathrm{C}_{30} \mathrm{H}_{43} \mathrm{FO}_{5} 502$.

The study was financially supported by the Russian Foundation of Basic Research (research project No. 17-00-00603a). The structural studies of compounds were carried out at the Center for Collective Use "Agidel" at the Institute of Petrochemistry and Catalysis, Russian Academy of Sciences.

\section{References}

(1) Dinan, L. Ecdysteroid structure-activity relationships. In: Atta-ur-Raman (ed) Studies in international products chemistry, Elsevier, Amsterdam, 2003, pp 3-71.

(2) Cymborowski, B. Bioassays for Ecdysteroids. In: Koolman J (ed) Ecdysone, from Chemistry to Mode of Action, Georg Thieme, Stuttgart, 1989, pp 144-149.

(3) Tomas, J.; Camps, F.; Coll, J.; Mele, E.; Pascual, N. Tetrahedron 1992, 48, 9809. doi: 10.1016/S0040-4020(01)81197-2.

(4) Lafont, R.; Dinan, L. J Insect Sci 2003, 3, 1. doi:10.1093/jis/3.1.7.

(5) Bathori, M.; Toth, N.; Hunyadi, A.; Marki, A.; Zador, E. Curr. Med. Chem. 2008, 15, 75. doi:10.2174/092986708783330674.

(6) Toth N.; Hunyadi, A.; Bathori, M.; Zador, E. Med. Chem. 2010, 17, 1974. doi:10.2174/092986710791163911.

(7) Martins, A.; Toth, N.; Vanyolos, A.; Beni, Z.; Zupko, I.; Molnar, J.; Bathori, M; Hunyadi, A. J. Med. Chem. 2012, 55, 5034. doi: 10.1021/jm300424n.

(8) Balazs, A.; Hunyadi, A.; Csabi, J.; Jedlinszki, N.; Martins, A.; Simon, A.; Toth, G. Magn. Reson. Chem. 2013, 51, 830. doi: 10.1002/mrc.4015.

(9) Martins, A.; Csabi, J.; Kitka, D.; Balazs, A.; Amaral, L.; Molnar, J.; Simon, A.; Toth, N.; Hunyadi, A. Molecules 2013, 18, 15255. doi:10.3390/molecules 181215255 . 
(10) Martins, A.; Sipos, P.; Der, K.; Csabi, J.; Miclos, W.; Verger, W.; Zalatnai, A.; Amaral, L.; Molnar, J.; Szabo-Revesz, P.; Hunyadi, A. BioMed Res. Int. 2015, 1. doi: org/10.1155/2015/895360.

(11) Prestwich, G.D. Pest. Managment Sci 1986, 17, 430-440. doi:10.1002/ps.2780170414.

(12) Cozzi, P.; Mongelli, A.; Suarato, A. Curr. Med. Chem. Anti-Cancer Agents 2004, 4, 93. doi:10.2174/1568011043482061.

(13) Singh, R.P.; Shreeve, J.M. Synthesis 2002, 17, 2561. doi:10.1055/s-200235626.

(14) Middleton, W. J. Org. Chem. 1975, 40, 574. doi:10.1021/jo00893a007.

(15) Csabi, J.; Martins, A.; Sinka, I.; Csorba, A.; Molnar, J.; Zupko, I.; Toth, G.; Tillekeratne, L.M.V.; Hunyadi, A. Med. Chem. Commun. 2016, 7, 2282. doi: 10.1039/C6MD00431H.

(16) Odinokov, V.N.; Savchenko, R.G.; Nazmeeva, S.R.; Galyautdinov, I.V.; Khalilov, L.M. Russ. J. Org. Chem. 2002, 38, 525. doi: 10.1023/A:1016547122573.

(17) Latypov, Sh.K.; Seco, J.M.; Quioa, E.; Riguera, R. J. Org. Chem. 1995, 60, 504. doi:10.1021/jo00108a008.

(18) Shellhamer, D.; Briggs, A.; Miller, B.; Prince, J.; Scott, D.; Heasley, V. J. Chem. Soc. Perkin Trans 2 1996, 973. doi:10.1039/P29960000973.

(19) Odinokov, V.N.; Galyatdinov, I.V.; Nedopekin, D.V.; Khalilov, L.M.; Shashkov, A.S.; Kachala, V.V.; Dinan, L.; Lafont, R. Insect Biochem. Mol. Biol. 2002, 32, 161. doi:10.1016/S0965-1748(01)00106-0. 